\title{
Die Septuaginta - Entstehung, Sprache, Geschichte
}

\author{
3. Internationale Fachtagung veranstaltet von Septuaginta Deutsch (LXX.D), \\ Wuppertal 22.-25. Juli 2010 \\ Hrsg. v. Siegfried Kreuzer, Martin Meiser u. Marcus Sigismund
}

Die Septuaginta Entstehung, Sprache, Geschichte

Herausgegeben von SIEGFRIED KREUZER MARTIN MEISER un MARCUS SIGISMUND

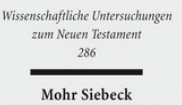

2012. XI, 757 Seiten. WUNT I 286

ISBN 978-3-16-152140-9

DOI 10.1628/978-3-16-152140-9

eBook PDF 189,00€

ISBN 978-3-16-151698-6

Leinen $189,00 €$
Die Septuaginta ist als jüdische Bibelübersetzung ab dem 3. Jh. v. Chr. entstanden. Sie wurde zur zentralen Grundlage des Judentums in der griechisch-hellenistischen Welt und in weiterer Folge für die Verbreitung des Christentums. Sie wurde von den meisten neutestamentlichen Autoren als Grundlage ihrer Schriftzitate benutzt und ist das Alte Testament in den orthodoxen Kirchen. Sie gibt Einblick in das Schriftverständnis und die Theologie des Griechisch sprechenden Judentums in der Antike. In textgeschichtlicher Hinsicht ist sie die wichtigste Quelle neben dem hebräisch-masoretischen Text, zumal sie, anders als die biblischen Texte aus Qumran, vollständig überliefert ist. Die Septuaginta-Forschung bildet einen eigenständigen Bereich neben der Erforschung der hebräischen Bibel und liegt im Schnittfeld mit dem Neuen Testament und mit antiker Geschichte und Religionsgeschichte. Der vorliegende Band enthält Studien zur Entstehung, zur Sprache, zur Geschichte sowie zur Wirkungsgeschichte der Septuaginta. Er gibt einen Überblick über den gegenwärtigen Forschungsstand und zeigt Perspektiven für weitere Forschung auf. Der Band ist erwachsen aus einer internationalen Tagung des Projektes Septuaginta Deutsch, die im Juli 2010 in Wuppertal stattfand. Mit Beiträgen von: Walter Ameling, Hans Ausloos, Walter Berschin, Eberhard Bons, John G. Cook, Cécile Dogniez, Patrick Egan, Helmut Engel SJ, Johannes Engels, Heinz-Josef Fabry, Marichiara Fincati, Katrin Hauspie, Valerie Kabergs, Martin Karrer, Jong-Hoon Kim, Wolfgang Kraus, Siegfried Kreuzer, Michael Labahn, Bénédicte Lemmelijn, Philippe LeMoigne, Johan Lust, Johannes Maglinao-Tromp, Martin Meiser, Mogens Müller, Wolfgang Orth, Melvin K. Peters, Ludger Schwienhorst-Schönberger, Marcus Sigismund, GertJ. Steyn, Emanuel Tov, Julio Trebolle Barrera, Frank Ueberschaer, Martin Vahrenhorst, Michael van der Meer, Anssi Voitila, Madeleine Wieger, Florian Wilk

Siegfried Kreuzer Geboren 1949; Promotion und Habilitation in Ev. Theologie; 1991-2015 Professor für Altes Testament an der Kirchlichen Hochschule in Wuppertal; seit 1999 Mitwirkung bei Septuaginta Deutsch und am Institut für Septuaginta und Biblische Textforschung.

Martin Meiser Born 1957; 1983-91 Lutheran minister in Bavaria; 1991-2001 Assistant for New Testament in Erlangen; 2001 05 Assistant for Early Church History in Mainz; 2005-07 Vicarious Professor in Münster/Westphalia; since 2007 Associate Professor in Saarbrücken.

Marcus Sigismund Geboren 1971; 2002 Dr. phil.; seit 1999 Lehrbeauftragter an der Bergischen Universität Wuppertal; seit 2007 Wissenschaftlicher Mitarbeiter am Institut für Septuaginta und biblische Textforschung, seit 2022 Koordinator der UniService Digitalisierung der Lehre an der Bergischen Universität Wuppertal. https://orcid.org/0000-0002-9345-767X

Jetzt bestellen:

https://mohrsiebeck.com/buch/die-septuaginta-entstehung-sprache-geschichte-9783161521409?no_cache=1 order@mohrsiebeck.com

Telefon: +49 (0)7071-923-17

Telefax: $+49(0) 7071-51104$ 